\title{
Public Health and Wild Fauna: Current Situation and Perspectives in Italy
}

\section{Massimo Giangaspero*}

Department of Veterinary Microbiology, Iwate University, Japan

The wild fauna represents an extraordinary added value of biodiversity. The environmental context, after a slow degradation, most linked to the logic of mere profit and indiscriminate industrial development, identify often the wild fauna as the bioindicator for nature and environment. Therefore, monitoring of wild fauna offers a wide source of relevant information. Taking into account that more than $60 \%$ of the newly identified infectious agents that have affected people over the past few decades have been caused by pathogens originating from animals or animal products, and of these zoonotic infections, $70 \%$ originated from wildlife, it is clear the importance of such information in the context of public health.

In Italy, the monitoring system of infectious diseases affecting wild fauna is ensured mainly by state experimental zoo prophylactic institutes, under the responsibility and supervision of the Department of Public Health, Food Safety and National Boards for Health Protection, Ministry of Health. Official data are collected by the National Reference Centre for Wild Animal Diseases (CeRMAS) and forwarded to the World Organization for Animal Health (Office International des Epizooties: OIE). The information on reported diseases is published through the World Animal Health Information System of OIE (WAHIS) (http://www.oie.int/wahis/).

Briefly, in 2011, a total of 3,467 cases of diseases affecting wild fauna have been notified from all the Italian Regions and the Autonomous Provinces of Trento and Bolzano. Largely preponderant, wild boars concerned the majority of the notifications, 2,710 (78.16\%), of which more than the $57 \%$ was reported from Emilia Romagna region. Hundred-sixty-nine cases (4.87\%) were reported from bovids (136 in chamois, 20 mouflon, 5 Pyrenean chamois, 4 ibex, 3 banteng, and 1 orix); 155 (4.47\%) were from cervids (140 in roe deer, 14 red deer, and 1 in Sardinian deer); 152 cases $(4.38 \%)$ were from avian species; 132 $(3.80 \%)$ were from canids (foxes and wolves); 98 (2.82\%) were reported from hares; 24 cases $(0.69 \%)$ were from reptilians; 13 cases $(0.37 \%)$ were from mustelids; 7 cases $(0.2 \%)$ were from various animal families (Tapiridae, Erinaceidae, Caviidae, Muridae, Myocastoridae, Istricidae); and 3 cases $(0.08 \%)$ were reported from marine mammals.

Virus diseases represented the half of the reported cases $(1,768$ $51 \%$ ), mainly porcine viruses (Aujeszky' disease virus, African swine fever virus, Circovirus). Canine distemper, mainly reported from the Autonomous Provinces of Trento and Bolzano, was the primary disease in canids e mustelids. Bacterial diseases represented the $20.7 \%$ (718) of notified cases: mainly salmonellosis and leptospirosis reported from wild boars from Lombardia region. Toxoplasmosis, mainly from wild boars from Emilia Romagna region, along with anaplasmosis, all cases from Lombardia region, mainly from roe deer and chamois, were the most frequent diseases caused by protozoa $(859-24.78 \%$ of notifications). Toxoplasmosis and anaplasmosis constituted the primary diseases in roe deer. Similarly, anaplasmosis was the most frequent disease reported in chamois. Trichinellosis only reported in canids, sarcoptic mange, mainly reported in foxes and chamois from north east regions, and echinococcosis constituted the $2.22 \%$ (77) of notifications. Chemical poisoning (45-1.3\%), due to pesticides and anticoagulants, was also reported mainly in avian species.

With concern to public health, the $44.5 \%$ of notifications were zoonosis ( 1,545 cases). Toxoplasmosis represented the most common zoonotic disease detected in 2011 in wild animals, with a total of 683 cases. Salmonellosis and leptospirosis were also diffuse, 282 and 199 cases, respectively. These diseases were mainly reported from wild boars. Similarly, all the 65 case of bovine tuberculosis have been reported in wild boars. This observed affinity was not the result of a higher number of samples collected from wild boars in confront of other animal species. In Valle d'Aosta region, out of about 700 samples, the majority (43\%) originated from roe deer, and samples from wild boar were the $14.28 \%$. However, notifications from wild boars were considerably higher than those reported from roe deer, $44.59 \%$ and $5.4 \%$, respectively. Mainly from Sardinia, from wild boars were also reported 28 cases of Brucellosi due to $B$. suis biovar 2, agent less pathogenic for humans in confront of other biovars. Furthermore, from wild boars were reported all the 23 cases of atypical mycobacterioses (M. avium, M. nonchromogenicum) generally considered non pathogenic, but among principal agents capable to cause bacteremia and diffuse infections in immune-compromised patients. From the same species, have been also reported 9 cases of infection due to Staphylococcus aureus, problematic for the production of thermostable toxins. These observations indicate that wild boar may have a non negligible potential negative role on public health.

One case of rabies in fox was reported from Veneto region. The disease deserves attention since during the previous year, in 2010; more than 200 foxes were killed by the virus in the northern east regions. Similarly, in 2011 only one case of echinococcosis was notified in wild boar. However, in Sardinia, this helmintic zoonotic disease shows a particularly serious epidemiology in human population, with 1,521 patients from 2001 to 2010. In the neighboring country of Switzerland, this disease is increasing since 2001. More frequently due to $E$. Multilocularis, in man from 2001 to 2008, cases increased of $150 \%$, frequently in urban areas, in relation with presence of foxes.

According to WAHIS (OIE), among zoo noses reported in human population in Italy in 2011, salmonellosis was the most common disease (539 cases). Emerging disease, West Nile fever (14 cases with 3 deaths) and campylobacteriosis (102 cases) were also reported. While in 2011, 11 notifications referred to West Nile fever in wild avian species, none concerned campylobacteriosis in wild fauna. Nevertheless, campylobacteriosis become the most important infectious disease reported in Europe in human population, with about 200,000 cases reported in 2009, according to European Centre for Disease Prevention

*Corresponding author: Massimo Giangaspero, Department of Veterinary Microbiology, School of Veterinary Medicine, Faculty of Agriculture, Iwate University, Japan, Fax: +81(0)19-621-6158; E-mail: giangasp@gmail.com

Received December 31, 2012; Accepted January 02, 2013; Published January 05, 2013

Citation: Giangaspero M (2013) Public Health and Wild Fauna: Current Situation and Perspectives in Italy. Clin Microbial 2: e107. doi:10.4172/2327-5073.1000e107

Copyright: ( 2013 Giangaspero M. This is an open-access article distributed under the terms of the Creative Commons Attribution License, which permits unrestricted use, distribution, and reproduction in any medium, provided the original author and source are credited. 
and Control. Transmission to man occurs through contact with animals and their products, mainly poultry meat, consumption of contaminated meat not sufficiently cooked. Contaminations often occur indirectly by the use of cutlery, dishes or other containers utilized for raw meat and reutilized for other food. The infection affects primarily infants under 4 years of age. A limited number of bacteria may determine severe abdominal pain and diarrhea. Symptoms are not limited to gastroenteritis but may vary affect different systems causing for example meningitis, endocarditis, osteomielitis, urinary infections or abortion. Mortality may also occur. In the Netherlands, in 200845 deaths have been reported from 3,340 cases, and in 2010 were 58 from 4,322 cases. These aspects indicate the need for an accurate monitoring of the pathogen also in wild fauna.

Among future perspectives currently considered by the Italian Ministry of Health, a national monitoring plan for detection of diseases affecting wild fauna is certainly the most important. The main objectives are surveillance, rapid alert for notifiable diseases (OIE listed diseases), and eventual application of containment measures. Training of veterinarians, definitions of competencies and harmonized surveillance at national level have been considered primary problems to be addressed. Zoonoses, diseases with health and economic impact on animal farming (animals and animal products trade restrictions) and diseases with impact on preservation of endangered species have been defined key words for a national plan. A number of diseases have been prioritized: rabies, classical and African swine fevers, Newcastle disease, Lyme, brucellosis and tuberculosis (only in case of occurrence of outbreaks in domestic animals), avian influenza and West Nile. Surveillance, passive or active, should be based on risk analysis, using local control plans, hunting activities and opportunistic sampling. Guidelines should be defined for transport of animals, sampling procedures, definition of specialized laboratories, case definition and validation of data. Collection of data should be enhanced through the implementation of an informatics system able to receive all information generated from surveillance and research activities: a wild fauna database. Concerning diagnostic activities, laboratories should be accreditated and diagnostic tests should be specifically validated for wild animal species.

In order to achieve the above mentioned objectives, in June 2012, the Department of Public Health, Food Safety and National Boards for Health Protection, Ministry of Health, established a technical working group on wild fauna, composed by representatives from the Ministry, the CeRMAS, the Italian Institute for Environmental Protection and Research (ISPRA), and the Italian society of Preventive Veterinary Medicine (SIMeVeP).

In conclusion, the establishment of a technical working group and the design of a national monitoring plan are fundamental bases and indicate institutional sensibility and the increasing attention for wild fauna. The choice of priorities requires the evaluation of several aspects to reach a common platform, and taking into account local realities. Zoonoses are fundamental part of monitoring plans in particular when concerning wildlife. The epidemiological data are essential elements and health professionals are called to develop it, applying reliable laboratory investigations. Finally, a correct and efficient monitoring will constitute the essential tool for the orientation and the application of activities, through an optimization of available resources, for the control and prevention in the field of animal health and public health. 